\title{
Doxazosin was associated with more stroke and cardiovascular disease events than chlorthalidone in high risk hypertension
}

\author{
The ALLHAT Officers and Coordinators for the ALLHAT Collaborative Research Group. Major cardiovascular events in \\ hypertensive patients randomized to doxazosin vs chlorthalidone. The Antihypertensive and Lipid-Lowering Treatment \\ to Prevent Heart Attack Trial (ALLHAT). JAMA 2000 Apr 19;283:1967-75.
}

QUESTION: In high risk hypertensive patients, is doxazosin (an $\alpha$-adrenergic blocker) or chlorthalidone (a diuretic) more effective in reducing cardiovascular disease (CVD) events?

Design

Randomised (allocation concealed*), blinded (clinicians and patients),* controlled trial with median 3.3 year follow up.

Setting

625 centres in the US and Canada.

\section{Patients}

24335 patients who were $\geqslant 55$ years of age (mean age 67 y, 53\% men, 49\% white non-Hispanic), had systolic blood pressure $(\mathrm{BP}) \geqslant 140 \mathrm{~mm} \mathrm{Hg}$ or diastolic $\mathrm{BP} \geqslant 90$ $\mathrm{mm} \mathrm{Hg}$, took medication for hypertension, and had $\geqslant 1$ other risk factor for coronary heart disease (CHD). \{Exclusion criteria included myocardial infarction (MI), stroke, or angina pectoris in the past 6 months; congestive heart failure (CHF) or ejection fraction $<35 \%$; or serum creatinine concentration $\geqslant 177 \mu \mathrm{mol} / \mathrm{l}\}.+97 \%$ of patients were included in the analysis.

\section{Intervention}

Patients were allocated to doxazosin, 2 to $8 \mathrm{mg}$ /day ( $\mathrm{n}=9067)$, or chlorthalidone, 12.5 to $25 \mathrm{mg} /$ day $(\mathrm{n}=15268)$.

\section{Main outcome measures}

The primary outcome was a composite end point of fatal CHD and non-fatal MI. Secondary outcomes were all cause mortality, combined CHD events (CHD death, non-fatal MI, revascularisation, and angina requiring admission to hospital), stroke, or combined CVD events (CHD death, non-fatal MI, stroke, revascularisation, angina, $\mathrm{CHF}$, and peripheral arterial disease).

\section{Main results}

Treatment with doxazosin was discontinued early. Analysis was by intention to treat. A proportional hazards model was used. At the stopping point, the groups did not differ for the primary outcome (relative risk [RR] $1.03,95 \% \mathrm{CI} 0.09$ to $1.17 ; \mathrm{p}=0.71$ ). However, for secondary outcomes, the doxazosin group had an increased risk for stroke (RR 1.19, 95\% CI 1.01 to 1.40; $\mathrm{p}=0.04)$ and combined CVD events (RR 1.25, CI 1.17 to $1.33 ; \mathrm{p}<0.001$ ), which included an increase in CHF (RR 2.04, CI 1.79 to $2.32 ; \mathrm{p}<0.001)$ and angina (RR 1.16 , CI 1.05 to $1.27 ; \mathrm{p}<0.001)$.

\section{Conclusion}

In high risk hypertensive patients, doxazosin was associated with a higher incidence of stroke and cardiovascular disease events, especially congestive heart failure, than was chlorthalidone.

*See glossary.

†Davis BR, Cutler JA, Gordon DJ, et al. Am J Hypertens 1996;9:342-60.

\section{COMMENTARY}

ALLHAT is a study of astonishing methodological beauty. A practice based trial, ALLHAT used both concealed allocation and double blinding in 625 centres; participants were randomised to one of several first line agents, doxazosin or chlorthalidone in this report; and the outcomes included all the major disease end points associated with uncontrolled hypertension. This study thus provides essential information about the optimal treatment strategy for patients with high BP.

Although chlorthalidone and doxazosin have a similar effect on BP lowering, they have different effects on the risks for stroke, angina, and CHF. In retrospect, it may seem obvious that drugs with such different mechanisms of action might well have different effects on various outcomes. The US Food and Drug Administration and other regulatory bodies, however, approve antihypertensive drugs on the basis of how well they lower BP. ${ }^{1}$ In light of the ALLHAT results, the assumption that the effect an antihypertensive agent has on $\mathrm{BP}$ lowering is a valid and adequate surrogate for the effect of the agent on the occurrence of major disease end points is no longer tenable. The criteria for antihypertensive drug approval ought to change.

The Sixth Report of the Joint National Committee on Prevention, Detection, Evaluation, and Treatment of High Blood Pressure recommended diuretics as first line agents but also included a section titled "May have favorable effects on comorbid conditions," which advocates the use of $\alpha$-blockers in patients with dyslipidemia or prostatism. ${ }^{2}$ Although heavily promoted, these "surrogate" arguments for favoring unproven antihypertensive treatments are not good evidence-based medicine. ${ }^{3}$ Despite beneficial effects on lipid concentrations, doxazosin was associated with an increased risk for CVD events. This study reinforces a key question for clinicians: if your patient with drug treated hypertension is not on a low dose diuretic, why not?

Bruce M Psaty, MD, PhD University of Washington Seattle, Washington, USA

Marco Pahor, MD

Wake Forest University Winston-Salem, North Carolina, USA

1 Psaty BM, Weiss NS, Furberg CD, et al.JAMA 1999;282:786-90 2 Arch Intern Med 1997:157:2413-46.

3 Psaty BM, Furberg CD. BMJ 1999;319:589-90. 